\title{
Poisson-like effect for flexural waves in periodically perforated thin plates
}

\author{
Penglin Gao, ${ }^{1, a)}$ José Sánchez-Dehesa, ${ }^{2}$ and Linzhi Wu ${ }^{1, b)}$ \\ ${ }^{1}$ Center for Composite Materials and Structures, School of Astronautics, Harbin Institute of Technology, \\ Harbin 150001, People's Republic of China \\ ${ }^{2}$ Wave Phenomena Group, Department of Electronic Engineering, Universitat Politècnica de València, \\ Camino de vera s.n. (Building 7F), ES-46022 Valencia, Spain
}

(Received 21 April 2018; revised 11 July 2018; accepted 9 August 2018; published online 29 August 2018)

\begin{abstract}
The Poisson-like effect, describing the redirection of waves by $90^{\circ}$, is shown to be feasible for flexural waves propagating in perforated thin plates. It is demonstrated that the lowest order symmetric leaky guided mode ( $\mathrm{S} 0$ mode) is responsible for the splitting of wave motion in two orthogonal directions. The S0 mode shows a feature of stationary waves containing standing wave modes in one and two orthogonal directions for smaller and larger holes, respectively. The former case is well understood thanks to the phenomenon of Wood's anomaly, which was first observed in optical gratings supposed to be transparent. On the contrary, the strong scattering caused by the larger holes leads to a mixed mode occurring when the incident wave is totally transmitted. The mixed mode easily couples with the incoming waves and, therefore, the Poisson-like effect activated under this mechanism is much stronger. Using the Poisson-like effect, a device is proposed in which about $82 \%$ of the incident mechanical energy is redirected to the perpendicular direction. Results obtained with arrays of free holes also apply to inclusions with parameters properly chosen. The findings may provide applications in beam splitting and waveguiding. (C) 2018 Acoustical Society of America. https://doi.org/10.1121/1.5051648
\end{abstract}

$[\mathrm{ANN}]$

Pages: $1053-1058$

\section{INTRODUCTION}

Redirection of waves is not a new concept. One typical example is provided at an interface separating two different dielectric media where light propagation is controlled by the well-known Snell's law. ${ }^{1}$ It is expected that, at normal incidence, the incident waves will pass through a flat interface directly without any deflection. By introducing abrupt phase changes on the interface, Yu et al. ${ }^{2}$ generalized the laws of reflection and refraction and achieved anomalous light propagation. This aspect has generated rising scientific interest and is developing into a prosperous research field, metasurfaces. ${ }^{3}$ Even though they provide new degrees of freedom to facilitate the manipulation of various types of waves, the redirection of normally incident waves to the perpendicular sides is still challenging.

Redirection of acoustic energy by $90^{\circ}$ has been obtained by constructing chains of cylindrical shells. ${ }^{4-6}$ By using arrays of perforated cylindrical shells, García-Chocano and Sánchez-Dehesa ${ }^{4}$ employed the strategy of wave redirection for the purpose of increasing the propagation path of sound and, thus, enhancing the dissipation loss of the structure. The redirection of acoustic waves was explained in terms of the

\footnotetext{
a) Also at: Wave Phenomena Group, Department of Electronic Engineering, Universitat Politècnica de València, Camino de vera s.n. (Building 7F), ES-46022 Valencia, Spain.

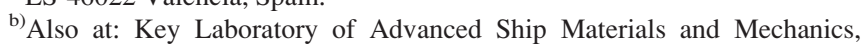
College of Aerospace and Civil Engineering, Harbin Engineering University, Harbin 150001, People's Republic of China. Electronic mail: wlz@hit.edu.cn
}

resonant excitation of a Wood's anomaly; i.e., a leaky guided mode propagating normally to the impinging direction. $^{7}$ Soon afterwards, Titovich and Norris ${ }^{5}$ introduced the term Poisson-like effect to describe the redirection of acoustic energy found in arrays of thin elastic shells embedded in water. A different mechanism explained this energy redirection, which has been proved to be possible by employing quadrupole resonance of cylindrical shells thanks to the dynamic coupling of normal unidirectional wave motion to the perpendicular one. More recently, Bozhko et al. ${ }^{6}$ further developed the microscopy theory of wave redirection applied to a linear chain of perforated shells in the air. They demonstrated that, at slightly oblique incidence, the sound waves can be redirected along a periodic chain to one single side provided the symmetric or antisymmetric eigenmode is resonantly excited.

This work expands the study of redirection of waves to the case of Lamb waves propagating in thin perforated plates. In such a system, the lower order transversely polarized plate wave modes dominate when the wavelength is much larger than the plate thickness. Particularly, our interest concentrates on the first order asymmetric Lamb mode, also termed as a flexural wave, whose behavior is well described by a biharmonic equation based on Kirchhoff-Love plate theory. ${ }^{8-10}$ By considering arrays of free holes we can adjust the scattering strength by merely changing the radius of the holes. It is shown later that the lowest order symmetric leaky guided mode (S0 mode) evolves with increasing radius, offering a new freedom to manipulate the propagation of flexural waves. Transmittance spectra and eigenfrequency analysis are the 
two main tools employed in the following discussions. On the one hand, the transmission properties of flexural waves through lattices of typical scatterers, like inclusions and free or clamped holes, have been obtained in the framework of the multiple scattering formalism. ${ }^{10,11}$ The accuracy of the semianalytical method has been fully demonstrated in the original works, therefore the relevant results will be directly used in this work without any repetition of details. On the other hand, due to the difficulties in solving transcendental equations with complex solutions, ${ }^{6}$ a commercial finite element package (COMSOL Multiphysics) is employed to conduct the eigenfrequency analysis in three-dimensional (3-D) solid mechanics module where full elastic equations are taken into consideration. However, in-plane motions are out of the scope of the present work. Therefore, only the out-of-plane motions will be discussed in the rest of the article.

\section{MODEL}

Let us consider a platonic crystal slab constructed in a homogeneous aluminum plate with parameters: Young's modulus $E=69 \mathrm{GPa}$, Poisson's ratio $\nu=0.33$, mass density $\rho=2.7 \times 10^{3} \mathrm{~kg} / \mathrm{m}^{3}$, and plate thickness $h=1 \mathrm{~mm}$. As shown in Fig. 1, the slab consists of three layers of free circular holes arranged in a square distribution with lattice constant $d=25 \mathrm{~mm}$. Moreover, $k_{p}$ is the wave number in the uniform host plate, $r$ and $\theta_{0}$ denote the radius of the holes and the incident angle of plane waves, respectively. In the eigenfrequency analysis, a row of holes are chosen as the unit cell of the infinite slab which serves as a leaky waveguide for flexural waves propagating along it. Perfectly matched layers (PML) are applied in the $x$-direction to simulate an infinite plate. In the $y$-direction, periodic boundary conditions are imposed on both sides of the unit cell. In this work, the reduced frequency, $k_{p} d / \pi$, is adopted since it provides useful information about the wavelength of the dispersive flexural waves. For example, two reduced units represent a wave with its wavelength being equal to the lattice constant; in absolute units, it corresponds to $15.54 \mathrm{kHz}$ for the parameters studied.

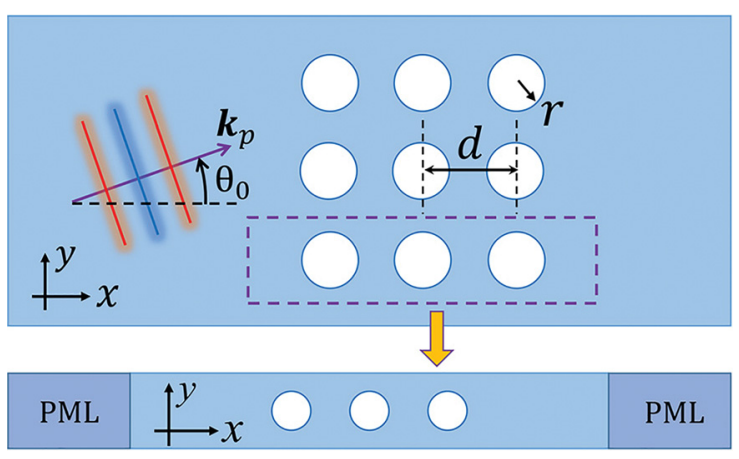

FIG. 1. (Color online) (Upper panel) Scheme of a platonic crystal slab consisting of three layers of free circular holes. The holes are distributed in a square lattice with period $d$. (Lower panel) Geometry of the finite element model employed to obtain the eigenfrequencies of the leaky-guided modes along the slab. Perfectly matched layers (PML) are employed at both ends.

\section{RESULTS AND DISCUSSION}

First, let us discuss the results for the case of weak scatterers. Figure 2 shows the dispersion relations of an infinite slab containing three layers of free holes with radius $r=3 \mathrm{~mm}$. Note that the dispersion relations are linear near the origin since we are representing the reduced frequency in the vertical axis. Only bands associated with the first three orders of out-of-plane eigenmodes are plotted here. Since the scattering of these small holes is relatively weak, it is observed that all the bands are nearly overlapping with that of an empty lattice represented by the solid lines. Nevertheless, the level repulsion originating from the impedance mismatch does indeed open a narrow bandgap near the diffraction limit, as it is seeing in the zoom views.

For a better understanding, Fig. 3 displays the eigenmodes of the out-of-plane displacement calculated at the edges of the narrow bandgap shown at $q d / \pi=0$. The nodal lines in the $y$-direction reflect the band folding effect, while the ones in the $x$-direction indicate the order of the mode. According to the symmetry with respect to the $x$ axis, these eigenmodes are classified into two categories: symmetric modes (S0, S1, and S2) and antisymmetric modes (A0, A1, and A2). Their corresponding eigenfrequencies are given in the caption of Fig. 3. Note that they are complex numbers with small positive imaginary parts, which determine the lifetime of the resonant modes. ${ }^{4}$ So, large imaginary part means short lifetime because more energy leaks from the guided mode to the free space. From another perspective, a

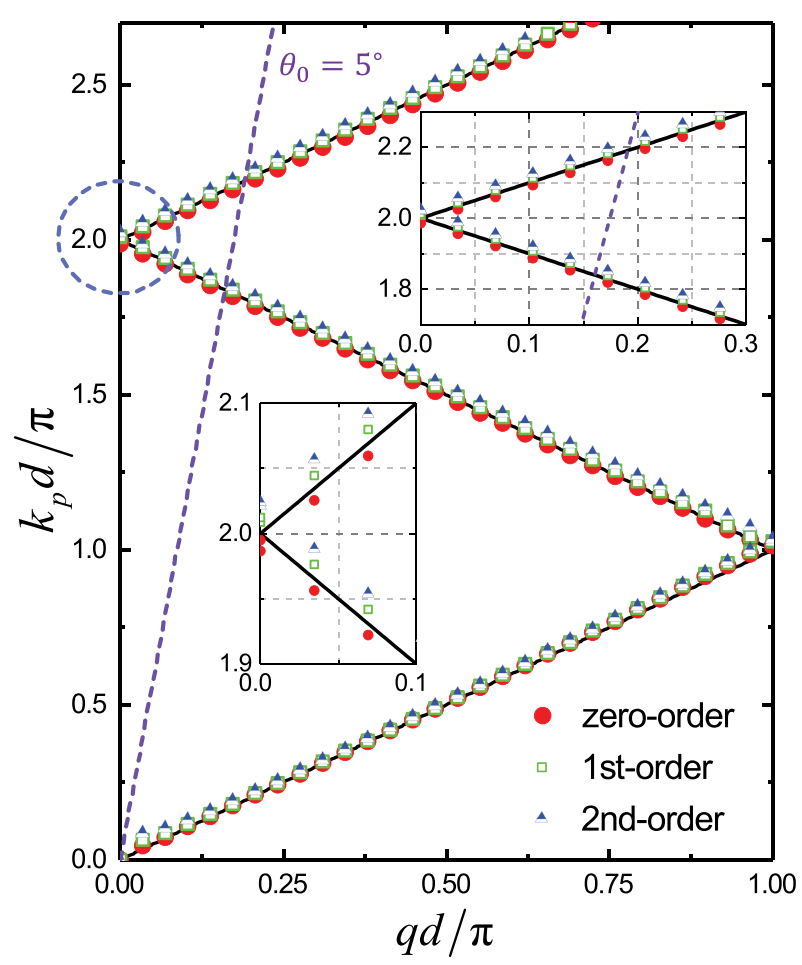

FIG. 2. (Color online) Band structures of an infinite slab containing three layers of free holes arranged in a square distribution with period $d$. The symbols represent the real parts of the calculated eigenfrequencies. The solid lines represent the dispersion relations of an empty lattice with the same periodicity $d$. Crossings of the dispersion relations with the dashed line give the frequencies of resonant coupling between the guided modes and the flexural wave with incident angle $\theta_{0}=5^{\circ}$. 


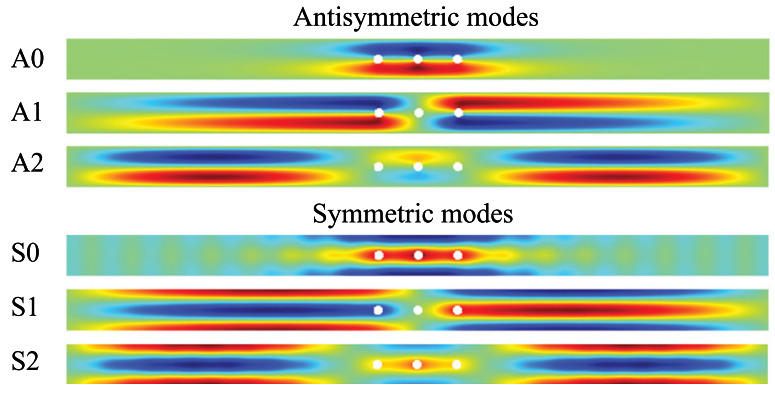

FIG. 3. (Color online) The first three orders of out-of-plane eigenmodes associated with the edges of the narrow bandgap at the high symmetry point $q d / \pi=0$. From top to bottom figures, the values of eigenfrequencies are $15125, \quad 15414+0.5 \mathrm{i}, \quad 15472+3.1 \mathrm{i}, \quad 15257+13.2 \mathrm{i}, \quad 15441+1.9 \mathrm{i}, \quad$ and $15478+6.0 \mathrm{i}$ (in $\mathrm{Hz}$ ), respectively.

leaky guided mode with short lifetime means more chances for the coupling with the incident waves. Except for the S0 mode, the rest of symmetric modes have negligible imaginary parts so that they cannot be excited at normal incidence. At slightly oblique incidence, however, both the lowest order symmetric and antisymmetric modes can be excited at frequencies predicted by the matching condition for the wave vectors: ${ }^{6}$

$$
k_{y}=k_{p} \sin \theta_{0}=q(f) .
$$

A case example is shown in Fig. 2 where the matching frequencies are given by the crossings points between the dispersion relations and the dashed line defining the incident angle $\theta_{0}=5^{\circ}$. Under this circumstance, incident waves with the lower frequency will be partially redirected along the negative $y$-axis while, for the upper frequency, the redirection will be along the positive $y$-axis (see the Appendix).

In summary, for weak scatterers, the previous results are consistent with the works reported for acoustic waves. ${ }^{4,6}$ What happens if we increase the radius of the holes? Figure 4(a) shows the transmittance spectra when a plane wave impinges on an infinite slab containing three layers of free holes. They correspond to holes with radii $3 \mathrm{~mm}$ (solid line), $5 \mathrm{~mm}$ (dashed

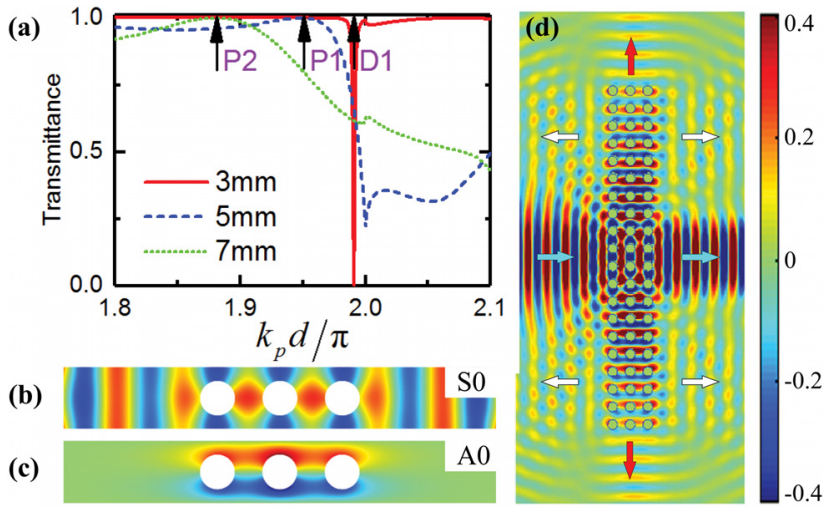

FIG. 4. (Color online) (a) Transmittance spectra for an infinite slab containing three layers of free holes whose radius increases from 3 and $5 \mathrm{~mm}$ to $7 \mathrm{~mm}$. The lowest order leaky guided modes S0 and A0 are displayed in (b) and (c), respectively, for the largest holes studied here. Their eigenfrequency values are $13547+1418.0 \mathrm{i}$ and 13395 (in $\mathrm{Hz}$ ), respectively. (d) Poisson-like effect excited at one peak frequency $(\mathrm{P} 2,13.75 \mathrm{kHz})$, where a Gaussian beam propagates from left to right. The arrows indicate the directions of the different waves entering or leaving the sample. line), and $7 \mathrm{~mm}$ (dotted line). It is observed that the transmission minimum, D1, evolves into broad transmission peaks, P1 and P2, when the radius increases. The narrow dip indicates the presence of a Wood's anomaly, ${ }^{7,12,13}$ which appears when the incident wave is strongly coupled to the S0 mode., ${ }^{4,14}$ This explanation applies to the transmission peaks as well. Nevertheless, we emphasize that in this case the S0 mode evolves to a mixed mode featured as stationary waves containing standing wave modes in two orthogonal directions, as shown in Fig. 4(b). In addition, we notice that, with the increasing radius, the dip and peak frequencies experience a red shift, which is predictable on account of the stronger level repulsion caused by the larger holes. During this process, the A0 mode [see Figs. 3 and 4(c)] is slightly changed, but it is always strongly localized and has no interest here.

The mixed eigenmode S0 shown in Fig. 4(b), with complex frequency $13.55+1.42 \mathrm{iHz}$, deserves additional discussion because of its large imaginary part. On the one hand, this property contributes to resonant coupling and facilitates its potential application for energy redirection. On the other hand, this property also implies large energy leakage into free space due to its extremely short lifetime. ${ }^{4,15}$ These features are clearly observed in Fig. 4(d), showing a snapshot of the out-of-plane displacement produced by the interaction of a Gaussian beam with frequency $13.75 \mathrm{kHz}$, corresponding to the transmission peak P2, with the slab of holes. The Poisson-like effect (vertical solid arrows) is clearly seen together with the energy leakage through the side of the slab (hollow arrows). Throughout this paper, the out-of-plane displacement snapshots are obtained via the multiple scattering algorithms developed in Ref. 10, which is not described here to avoid redundancy. This frequency value agrees fairly well with the eigenfrequency of the mixed mode S0 independently calculated with a commercial 3-D finite element method (FEM).

The leaky behavior of the fundamental eigenmode $\mathrm{S} 0$ is significantly influenced by the radius of the holes. For comparison, Fig. 5 plots the calculated sideways transmissions, $T_{\text {side, }}$ of several finite slabs being three layers thick. Here, $T_{\text {side }}$ is defined as the energy ratio of the redirected waves to the incident ones, $T_{\text {side }}=2 E_{\text {top }} / E_{\text {inc }}$, where the energy-related quantities are numerically calculated by integrating the energy flux over the boundaries of the finite slab., ${ }^{5,17}$ The

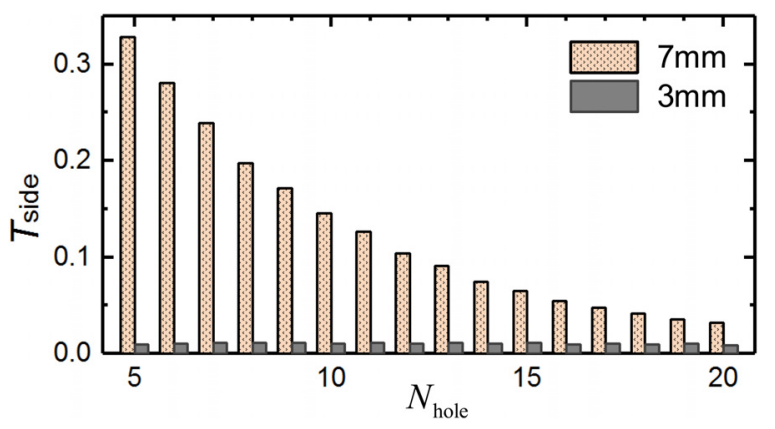

FIG. 5. (Color online) The dependence of sideways transmission, $T_{\text {side }}$ on the length of a slab containing three layers of small/large holes. The length varies with the number of holes in each column $N_{\text {hole }}$, and Fig. 4(d) provides an example with $N_{\text {hole }}=20$. 
dependence of $T_{\text {side }}$ with the number of holes along the $y$ direction $N_{\text {hole }}$ is analyzed. It is observed that the Poisson-like effect is strongly enhanced when the finite slab is constructed with the larger holes. In addition, as shown in Fig. 4(b), the eigenfrequencies values are complex and large imaginary parts mean more energy leakage from the guided mode to the free space due to the complex propagation wavenumber. ${ }^{4,15}$ As a result, the energy transferred to the mixed mode decays more quickly compared with the other case. This effect explains the failure in splitting bi-frequency signals ${ }^{6}$ at oblique incidence; the energy coupled to the symmetric mode is completely leaked out (see the Appendix).

From Fig. 5, we conclude that fewer holes are preferred in each column if one wants to redirect more energy to the perpendicular sides. In addition, some other improvements can be made to further increase the sideways transmission. Here, we have considered a platonic crystal barrier placed at the rear of the finite slab in order to totally reflect the waves transmitted through the slab. The barrier consists of three layers of two-beam resonators. ${ }^{9}$ There are two main reasons justifying the choice of this type of resonators. First, the resonance induced bandgap can be easily tuned to specific working frequencies by changing the geometric parameters. Second, two beams contribute to bandgap enhancement when they are arranged in parallel with the wavefront of the incident waves. ${ }^{10,16}$ The geometric parameters described in Fig. 6(a) are $R_{1}=4 \mathrm{~mm}, R_{2}=7 \mathrm{~mm}, b=2 \mathrm{~mm}$, and $l=20 \mathrm{~mm}$. The band structure shown in Fig. 6(b) indicates that the propagation of flexural waves is completely (a)

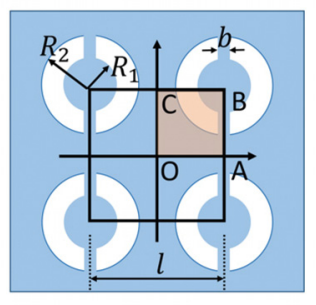

(b)

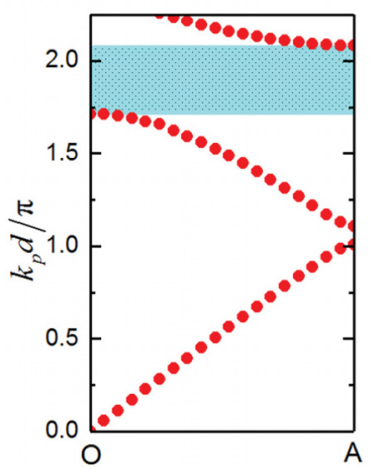

(c)

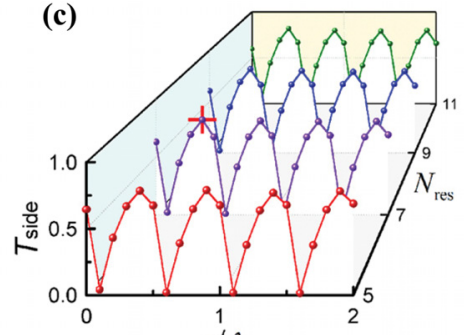

(d)

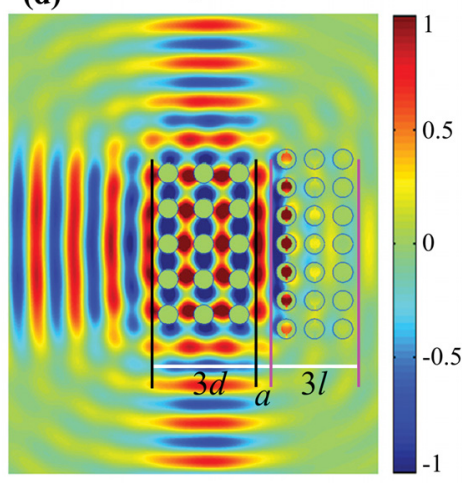

FIG. 6. (Color online) (a) Scheme of lattice of two-beam resonators in real space together with the definitions of the high symmetry directions in reciprocal space, where the shadowed region represents the irreducible Brillouin zone. (b) Band structure of the flexural waves propagating along the OA direction. (c) Optimization process for a barrier in order to increase the sideways transmission, where $N_{\text {res }}$ represents the number of two-beam resonators in each column, and $a$ denotes the spacing between the barrier and slab. (d) Poisson-like effect excited by a Gaussian beam at one peak frequency $(\mathrm{P} 2,13.75 \mathrm{kHz})$, where an optimized barrier [see the cross in figure (c)] is placed right behind the slab. prohibited in the frequency region between 11.42 and $16.79 \mathrm{kHz}$. In reduced units, the lower and upper bounds are 1.72 and 2.08, respectively. Figure 6(c) studies the influence of the number of two-beam resonators in each column, $N_{\text {res }}$, and the spacing $a$ between the slab and barrier on the sideways transmission, $T_{\text {side. }}$. Due to the destructive and constructive interference $T_{\text {side }}$ changes periodically with $a$, while $N_{\text {res }}$ has little influence on it. At the position marked with a cross symbol in Fig. 6(c) the sideways transmission reaches $82 \%$, which is about 25 times larger than that obtained in Fig. 4(d). This position defines the optimum parameters to design a device showing the strongest Poisonlike effect. Figure 6(d) shows a snapshot of the out-of-plane displacement pattern obtained from a multiple scattering simulation of the optimum device.

For the sake of comprehensiveness, in what follows we briefly discuss the performance of slabs based on other kinds of scatterers, such as clamped holes and inclusions. Let us stress that the mixed S0 eigenmode and holes with clamped boundaries are incompatible and, therefore, it is impossible to redirect flexural waves with platonic crystal slabs containing clamped holes. However, inclusions with properly chosen parameters are likely to work since the void in the cavities can be considered as an extremely soft material without mass and modulus. For demonstration, two kinds of inclusions are considered here, and their physical parameters are: $E=102 \mathrm{GPa}, \nu=0.30, \rho=4.5 \times 10^{3} \mathrm{~kg} / \mathrm{m}^{3}$ (titanium); $E=16 \mathrm{GPa}, \nu=0.44, \rho=11.3 \times 10^{3} \mathrm{~kg} / \mathrm{m}^{3}$ (lead). Figure 7 provides the transmittance spectra and $\mathrm{S} 0$ eigenmodes for an infinite slab containing three layers of inclusions with the same radius $r=7 \mathrm{~mm}$. These results have been obtained following a procedure similar to that employed in Fig. 4. The real parts of the complex eigenfrequencies obtained using FEM agree fairly well with the frequencies of the peak (P1) and the narrow minimum (D1) in the transmission spectra. The transmittance profile indicates that titanium (lead) inclusions behave like weak (strong) scatterers. In the latter case, the huge impedance mismatch leads to a stronger level repulsion so that the resonant frequency shifts to a much lower value, which provides a new degree of freedom to control the Poisson-like effect.

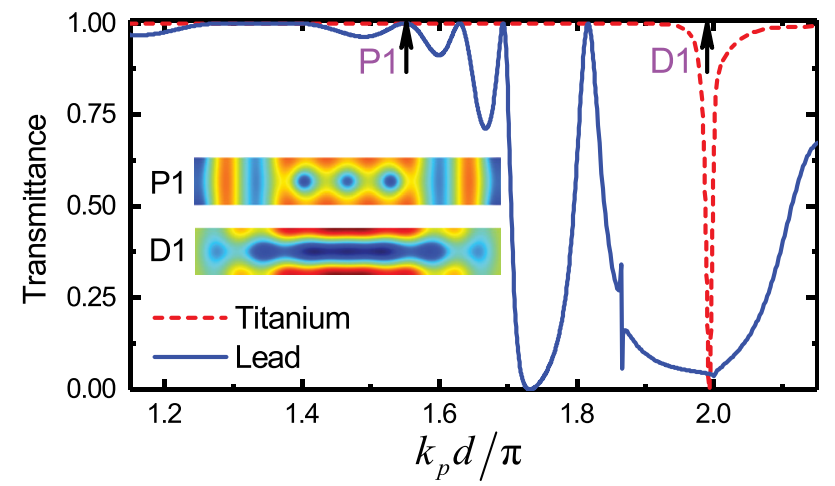

FIG. 7. (Color online) Transmittance spectra for an infinite slab containing three layers of inclusions. The insets show the S0 modes for the two kinds of inclusions considered here. Their eigenfrequencies are $15284+111.7 \mathrm{i}$ and $9533+816.0 \mathrm{i}$ (in $\mathrm{Hz}$ ) for slabs containing titanium and lead inclusions, respectively. In reduced units, the real parts correspond to 1.98 and 1.57 , respectively. 


\section{SUMMARY}

In conclusion, the Poisson-like effect has been demonstrated for flexural waves interacting with finite platonic crystal slabs. The effect arises because of the coupling properties of the fundamental symmetric leaky guided mode embedded in the slab. For weak scatterers, like small free holes or inclusions with materials similar to the background, the energy redirection is explained in term of a Wood's anomaly. The redirection appears as a minimum in the transmittance spectrum, indicating the excitation of the S0 leaky eigenmode. For strong scatterers, like large free holes or inclusions with a large impedance mismatch with the background, the S0 eigenmode is a mixed mode with a complex eigenfrequency of considerable large imaginary part. In this case, the incident wave is totally transmitted. Using this property, an optimum structure is here designed in which the Poisson-like effect is greatly enhanced. The structure consists of a finite platonic crystal slab with a barrier for flexural waves at its rear face. Numerical simulation shows that about $82 \%$ of the incident energy is redirected to the perpendicular directions. At oblique incidence, where both the symmetric and antisymmetric modes can be resonantly excited at different but close frequencies, we have obtained that the redirection performance is slightly different for weak and strong scatterers. The results here reported have potential applications in designing practical devices for beam splitting and waveguiding in thin plates.

\section{ACKNOWLEDGMENTS}

Work supported by the Ministerio de Economía y Competitividad of Spain and the EU Fondo Europeo de Desarrollo Regional under Project No. TEC2014-53088-C3-1$\mathrm{R}$, and the National Natural Science Foundation of China under Grants Nos. 11432004 and 11421091. P.G. acknowledges a scholarship with No. 201606120070 provided by China Scholarship Council.

\section{APPENDIX: ENERGY REDIRECTION AT OBLIQUE INCIDENCE}

At normal incidence $\theta_{0}=0^{\circ}$, only the lowest order symmetric mode ( $\mathrm{S} 0$ mode) can be excited by the incident

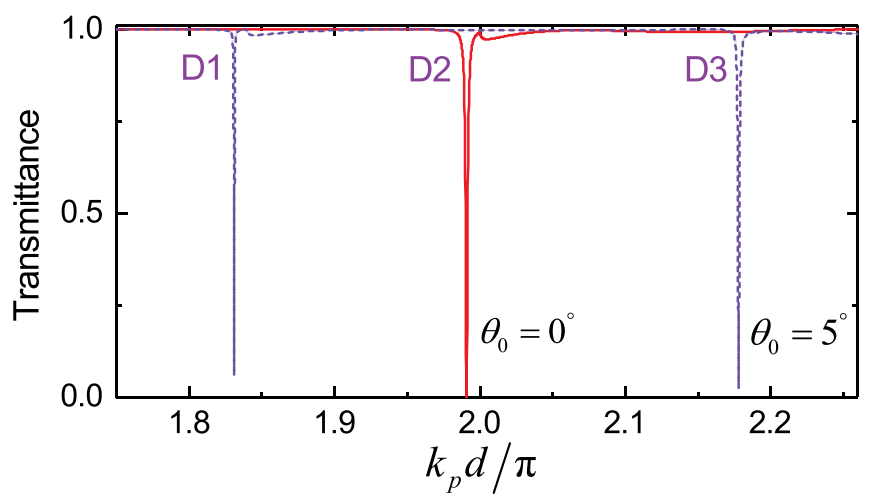

FIG. 8. (Color online) Transmittance spectra for an infinite slab containing three layers of free holes with radius $r=3 \mathrm{~mm}$.
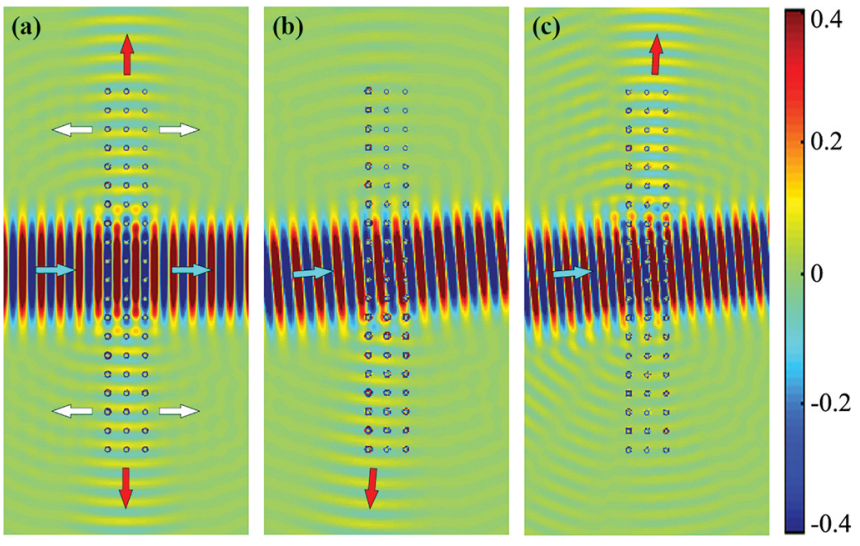

FIG. 9. (Color online) Redirection of flexural waves through a platonic crystal slab containing $3 \times 20$ free circular holes $(r=3 \mathrm{~mm})$. The systems in (a), (b), and (c) are excited by a Gaussian beam having frequencies extracted at D2, D1, and D3 (see Fig. 8), respectively. The incident waves propagate from left to right. The vertical solid arrows indicate the propagation directions of the redirected waves leaving the sample while the arrows indicate the energy leakage through the lateral sides of the slab.

flexural waves. Nevertheless, it is emphasized that, thanks to symmetry broken, the coupling of incident waves to the antisymmetric modes is possible at slightly oblique incidence provided the matching condition is satisfied. ${ }^{6}$ To prove this, Fig. 8 shows the transmittance spectra for an infinite slab containing three layers of free circular holes with radius $r=3 \mathrm{~mm}$, where the solid and dotted lines represent the results for two incident angles $\theta_{0}=0^{\circ}$ and $5^{\circ}$, respectively. The transmission dips indicate the excitation of both symmetric and antisymmetric modes. At oblique incidence, the dips come into being at 1.83 and 2.18 in reduced units. The values agree well with those predicted by the matching condition (see the crossings in Fig. 2). Since the higher order modes have eigenfrequencies of negligible imaginary parts, only the lowest order leaky guided modes are excited for the system studied here, which is a little different from the results reported for acoustic waves. ${ }^{4}$

Through the resonant coupling, it is possible to redirect flexural waves to the orthogonal directions. ${ }^{6}$ Fig. 9 shows the numerical simulations for the system excited by a Gaussian beam. At normal incidence [see Fig. 9(a)], part of the incident energy is equally redirected to the two perpendicular sides, and this behavior has been termed as Poisson-

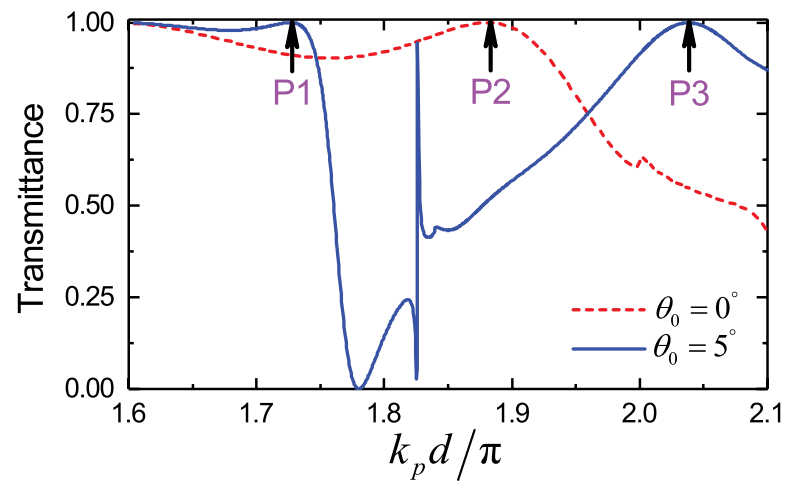

FIG. 10. (Color online) Transmittance spectra for an infinite slab containing three layers of free holes with radius $r=7 \mathrm{~mm}$. 


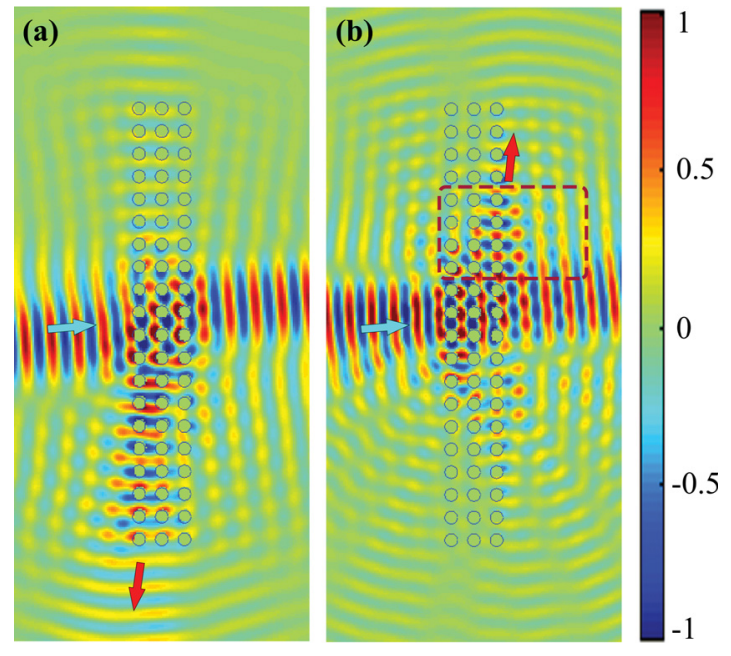

FIG. 11. (Color online) Redirection of flexural waves through a platonic crystal slab containing $3 \times 20$ free circular holes $(r=7 \mathrm{~mm})$. The out-ofplane displacement fields in (a) and (b) are obtained by considering a Gaussian beam having frequencies extracted at P1 and P3 (see Fig. 10), respectively. The incident waves propagate from left to right, and the vertical arrows indicate the propagation directions of the redirected waves leaving the sample.

like effect. ${ }^{5}$ At slightly oblique incidence [see Figs. 9(b) and 9(c)], however, the energy transferred to the A0 and S0 modes will be redirected downward and upward individually. The behaviors are the same as those reported in Refs. 4 and 6 where the perforated shells serve as weak scatterers for airborne acoustic waves.

Previous analysis applies to periodic structures containing larger holes as well, but instead of Wood's anomaly, it is the broad transmission peak that indicates the resonant coupling. Figure 10 shows the transmittance spectra for an infinite slab containing three layers of larger holes $r=7 \mathrm{~mm}$, where two peaks $\mathrm{P} 1$ and $\mathrm{P} 3$ are observed at oblique incidence. As has been performed in Figs. 9(b) and 9(c), Fig. 11 provides the out-of-plane displacement maps excited at the resonant frequencies. Due to the anomalous scattering, it is observed that the energy coupled to the A0 mode is redirected to the lower end [see Fig. 11(a)], while the energy transferred to the S0 mode leaks out completely before it was redirected to the upper end [see Fig. 11(b)].

${ }^{1}$ M. Born and E. Wolf, Principles of Optics: Electromagnetic Theory of Propagation, Interference and Diffraction of Light (Elsevier, New York, 2013), Chap. 1.

${ }^{2}$ N. Yu, P. Genevet, M. A. Kats, F. Aieta, J. P. Tetienne, F. Capasso, and Z. Gaburro, "Light propagation with phase discontinuities: Generalized laws of reflection and refraction," Science 334, 333-337 (2011).

${ }^{3}$ H. Chen, A. J. Taylor, and N. Yu, "A review of metasurfaces: Physics and applications," Rep. Prog. Phys. 79, 076401 (2016).

${ }^{4}$ V. M. García-Chocano and J. Sánchez-Dehesa, "Anomalous sound absorption in lattices of cylindrical perforated shells," Appl. Phys. Lett. 106, 124104 (2015).

${ }^{5}$ A. Titovich and A. N. Norris, "Acoustic Poisson-like effect in periodic structures," J. Acoust. Soc. Am. 139, 3353-3356 (2016).

${ }^{6}$ A. Bozhko, J. Sánchez-Dehesa, F. Cervera, and A. Krokhin, "Redirection and splitting of sound waves by a periodic chain of thin perforated cylindrical shells," Phys. Rev. Appl. 7, 064034 (2017).

${ }^{7}$ R. W. Wood, "XLII. On a remarkable case of uneven distribution of light in a diffraction grating spectrum," Philos. Mag. 4, 396-402 (1902).

${ }^{8}$ K. F. Graff, Wave Motion in Elastic Solids (Courier Corporation, New York, 2012), Chaps. 3 and 4.

${ }^{9}$ A. Climente, P. Gao, L. Wu, and J. Sánchez-Dehesa, "Scattering of flexural waves from an $N$-beam resonator in a thin plate," J. Acoust. Soc. Am. 142, 3205-3215 (2017).

${ }^{10} \mathrm{P}$. Gao, A. Climente, J. Sánchez-Dehesa, and L. Wu, "Theoretical study of platonic crystals with periodically structured $N$-beam resonators," J. Appl. Phys. 123, 091707 (2018).

${ }^{11}$ L. Cai and S. A. Hambric, "Multiple scattering of flexural waves on thin plates," J. Vib. Acoust. 138, 011009 (2015).

${ }^{12}$ Lord Rayleigh, "On the dynamical theory of gratings," Proc. R. Soc. London A 79, 399-416 (1907).

${ }^{13}$ U. Fano, "The theory of anomalous diffraction gratings and of quasistationary waves on metallic surfaces (Sommerfeld's waves), J. Opt. Soc. Am. 31, 213-222 (1941).

${ }^{14}$ R. P. Moiseyenko, Y. Pennec, R. Marchal, B. Bonello, and B. DjafariRouhani, "Broadband attenuation of Lamb waves through a periodic array of thin rectangular junctions," Phys. Rev. B 90, 134307 (2014).

${ }^{15}$ A. Ip and D. R. Jackson, "Radiation from cylindrical leaky waves," IEEE Trans. Antennas Propag. 38, 482-488 (1990).

${ }^{16} \mathrm{E}$. Andreassen, K. Manktelow, and M. Ruzzene, "Directional bending wave propagation in periodically perforated plates," J. Sound Vib. 335, 187-203 (2015).

${ }^{17}$ A. Norris and C. Vemula, "Scattering of flexural waves on thin plates," J. Sound Vib. 181, 115-125 (1995). 